\title{
IVD. 14 - Rapid Colorimetric Detection of Zika Virus from Mosquito Samples by Reverse Transcription Loop-Mediated Isothermal Amplification (RT-LAMP)
}

Severino Jefferson Ribeiro da Silva ${ }^{1 \star}$; Marcelo Henrique Santos Paiva ${ }^{1}$; Duschinka Ribeiro Duarte Guedes ${ }^{1}$; Larissa Krokovsky ${ }^{1}$; Fábio Lopes de Melo ${ }^{1}$; Constância Flávia Junqueira Ayres $^{1}$; Lindomar José Pena ${ }^{1}$.

1 Fiocruz - Fundação Oswaldo Cruz.

Introduction: The rapid spread of Zika virus (ZIKV) represents a global public health problem, especially in countries that circulate several vector mosquito vectors and favorable conditions for virus transmission, such as Brazil. In these areas, improvement in mosquito control needs to be a top priority, but mosquito viral surveillance occurs inefficiently in endemic countries. Currently, the reverse transcriptase reaction followed by quantitative polymerase chain reaction (qRT-PCR) is the gold standard for molecular diagnostic of ZIKV in mosquito samples. However, the technique presents high cost and limitations for Point-of-care (POC) diagnostics, which hampers its application for a large number of samples in entomological surveillance programs in low-resource areas. In view of this scenario, there remains the need to develop new POC diagnostics.

Objective: In this context, the aim of this work was to develop and validate a diagnostic platform based on the reverse transcriptase technique followed by isothermal loop-mediated amplification (RT-LAMP) for detection of ZIKV in mosquito samples.

Methodology: Initially, was determined the ability of RT-LAMP to detect ZIKV in Aedes aegypti under controlled conditions, including experimentally infected female A. aegypti mosquitoes. In addition, the specificity were assayed by testing the cross-reactivity with other arboviruses currently circulating in Brazil, including ZIKV (PE243), DENV-1 (PE/97-42735), DENV-2 (PE/95-3808), DENV-3 (PE/02-95016), DENV-4 (PE/10-0081), YFV (17DD) and CHIKV (PE2016-480). To evaluate the analytical sensitivity, ZIKV strain PE243 was 10-fold serially diluted in crude lysates of uninfected $A$. aegypti mosquito. After dilution, samples were directly assayed by RT-LAMP without RNA isolation. Subsequently, the validation of the RT-LAMP test was performed with 60 samples of A. aegypti and Culex quinquefasciatus mosquitoes, including samples of laboratory-infected mosquitoes and naturally infected mosquitoes collected in the metropolitan region of Recife-PE. Lastly, the value per reaction was calculated based on the cost of all the reagents.

Results: Regarding the results, the RT-LAMP assay was highly specific for detection of ZIKV in 20 minutes and was up to 10,000 times more sensitive than qRT-PCR for detection of ZIKV in mosquito samples. The RT-LAMP had a sensitivity of $100 \%$, specificity of $91.18 \%$, and overall accuracy of $95.24 \%$, highlighting the potential of RT-LAMP for detection of ZIKV. As for the cost of each reaction of the RT-LAMP, the value was one real ( $\mathrm{R} \$ 1.00)$.

Conclusion: We have developed a low cost, POC diagnostic platform based on the RT-LAMP assay to detect ZIKV in mosquito samples collected at the epicenter of the Zika epidemics in Brazil. The test is a robust, fast and inexpensive tool for surveillance of ZIKV in mosquito populations and will enable developing countries to establish better viral surveillance in vectors and improve the efficacy of control programs. Our results provide a potential new straightforward and molecular diagnostic test for ZIKV in arthropod vectors.

Keywords: Diagnostic; Point-of-care; ZIKV 